\title{
Ein neues Fragment des ,Rennewart Ulrichs von Türheim in Heringen (Helme)
}

\author{
Jessica Bruns $\cdot$ Christian Speer
}

$\mathrm{I}$ m Zuge der systematischen Erfassung der mittelalterlichen und frühneuzeitlichen Stadtbücher Deutschlands werden durch die Mitarbeiter des DFG-Langfristvorhabens ,Index Librorum Civitatum $^{{ }^{\prime}}$ gelegentlich interessante Funde von makulierten Inkunabeln und Handschriften gemacht, die sie an die entsprechenden Stellen bzw. spezialisierte Kolleginnen und Kollegen weiterleiten. ${ }^{2}$ Im Fall des ,Rennewart'-Fundes von Heringen (Helme) haben sich die Entdecker entschieden, diesen selbst bekannt zu machen. Die Vorgeschichte des Fundes soll nur kurz umrissen werden: Aus der Literatur ${ }^{3}$ vermuteten sie, dass das Stadtarchiv Heringen (Helme) für das eingangs genannte Stadtbuchprojekt interessante Handschriften besitzen könnte, jedoch existierten keine gedruckten Findmittel. Bis zum Anfang der I990er Jahre wurden die Bestände des Stadtarchivs Helme im Kreisarchiv Nordhausen verwahrt. Danach wurden sie auf Wunsch der Stadt wieder in die eigene Obhut genommen, allerdings gab es vor Ort weder adäquate Archivräume noch Personal, das sich hauptamtlich um die Archivalien hätte kümmern können. Das Archivgut wurde daher auf dem ausgebauten Dachboden der alten Schule aufgestellt. Nach einer ersten Kontaktaufnahme mit der Stadt im Sommer 20I7 und hartnäckig geführten Anfragen konnten wir schließlich im Februar 2019 nach Heringen (Helme) reisen und die Bestände in Augenschein nehmen. Da anstelle eines Findbuchs im engeren Sinn lediglich ein Karteikarteninventar existierte, war es notwendig, alle Karteikarten durchzugehen und potenziell einschlägige Archivalien im Original einzusehen. $\mathrm{Zu}$ den aus Sicht des Projektes besonders relevanten Quellen gehörten dabei unter anderem 66 Bände, die als „Rechnungen des Raths zu Heringen und Manuale über Einnahme und Ausgabe" bezeichnet wurden und eine Laufzeit von 1600 bis 1764 aufwiesen. Eine genauere Prüfung der Bände ergab, dass in der ersten Hälfte des 20. Jahrhunderts offensichtlich auf Betreiben des damals für den Kreis Sangerhausen zuständigen Archivpflegers Hermann Hiller mehrere Rechnungsjahrgänge zu Sammelbänden vereinigt und mit zeitgenössischen Leineneinbänden versehen worden waren. ${ }^{4}$ Zwischen den einzelnen Heften fanden sich immer wieder ältere Pergamentblätter, die als Einbände der ursprünglichen Einzelrechnungen gedient hatten. Zumeist handelte es sich dabei um liturgische Handschriften wie Hymnarien oder Gradualien. Im Band mit der Signatur XV.4 wurde jedoch ein hinter dem Buchblock liegendes, loses Pergamentblatt aufgefunden, das nicht mit einem lateinischen, sondern einem mittelhochdeutschen Text beschriftet war, wobei der Name Willebalm sofort ins Auge fiel. Eine anschließende Textbestimmung ergab, dass es sich bei dem aufgefundenen Fragment um eine Textpassage aus dem ,Rennewart' Ulrichs von Türheim handelte. ${ }^{5}$

Das Heringer Fragment besteht aus dem unteren Teil eines Pergamentblattes, das beidseitig mit Text beschrieben worden ist (Abb. I und 2). Augenscheinlich ist das Blatt zu Beginn des i7. Jahrhunderts als Einband für einen Jahrgang der Rechnungen des Rats der Stadt Heringen (Helme) wiederverwendet worden, denn auf ihm findet sich die Aufschrift „Rathsrechnung Von Mich(aelis) A(nn)o I6o5 bijs Mich(aelis) A(nn)o 1606". Der Titel stammt dabei von derselben Hand wie die Rechnungen. Durch Falzen 
der Ränder wurde das Fragmentblatt auf das Format der Rechnungen angepasst. An der entsprechenden Stelle des Rechnungsheftes sowie auf dem Fragment selbst lassen sich noch Spuren der ursprünglichen Verklebung erkennen. Das Rechnungsheft muss später einen Wasserschaden mit anschließendem Schimmelbefall erlitten haben, infolgedessen der schmale Rücken sowie Teile des Umschlagblattes stark beschädigt wurden und schließlich die Rückseite des Umschlages abriss und verlorenging. Als man im 20. Jahrhundert die Einzeljahrgänge der Rechnungshefte zu Sammelbänden vereinte, hat man sich dann nicht die Mühe gemacht, die ursprünglichen Einbände zu entfernen, sondern die Hefte gesamthaft mit einem neuen Einband versehen. Das ursprünglich als Umschlag genutzte ,Rennwart'-Fragment wurde dabei in den neu geschaffenen Sammelband eingelegt.

Der Text des Fragments ist in zwei Spalten geschrieben, wobei die Beschreibräume vorab durch dünne Linien begrenzt worden sind. Am unteren Blattrand wurde ein breiter Rand von $52 \mathrm{~mm}$ gelassen. In der Breite misst das Fragment $202 \mathrm{~mm}-$ nimmt man die Umbüge hinzu, sind es $235 \mathrm{~mm}$. An der höchsten Stelle ist der Blattrest ca. $150 \mathrm{~mm}$ hoch. Die beiden Spalten sind jeweils $80 \mathrm{~mm}$ breit. Der Leerraum dazwischen bemisst sich auf $13 \mathrm{~mm}$. Die Zeilen selbst haben eine Höhe von $7 \mathrm{~mm}$. Das Pergament ist von mittlerer Qualität. Ein Riss im Beschreibstoff, der - wie entsprechende Nadellöcher belegen - in früherer Zeit vernäht gewesen war, wurde vom Schreiber umschrieben. Die Versanfänge sind im Unterschied zu vielen anderen bekannten ,Rennewart'-Fragmenten nicht abgesetzt und es finden sich auch keine Rubrizierungen. Als Schmuck- bzw. Gliederungselemente sind einzig zwei zweizeilige rote Lombarden zu nennen.

Mehr oder minder vollständig überliefert geblieben sind jeweils die unteren I4 Zeilen einer jeden Spalte. Ausgehend von der Edition des ,Rennewart', die Alfred Hübner 1938 herausgegeben hat, umfasst das Heringer Fragment die folgenden Verse: Rectoseite 21015-21028 und
2I053-2I066, Versoseite 2109I-2IIO4 sowie 2II272II40. ${ }^{6}$ Inhaltlich handelt es sich um Ausschnitte des Gesprächs, das Willehalm mit Malefer, Lois und seiner Schwester, der Königin, ungefähr zur Werkmitte führt, als Malefer bereits auf die Seite der Christen übergetreten ist. Willehalm hat erfahren, dass Terramer einen neuen Angriff plane und ist deshalb zu König Lois gereist, um dessen Hilfe zu erbitten. Im Laufe des Gesprächs sichern schließlich Lois und Malefer ihre Unterstützung zu und die Königin verspricht, Gold für die Rekrutierung von Söldnern bereitzustellen. Der Text des Fragments lautet wie folgt:

\section{Rectoseite:}

21015 Terremer wil vber mer Mit eyme alfo grozen her daz er grozer nie gevůrte Sint mich fin haz gerůrte Dar geyn min vrochte ift nicht ringe

21020 Er bot mir daz im bringe Eyn kvining heyzet kyron vnde ift von lacedemon Starker rifen wol dritzich vnde daz terremer fi vlitzich 21025 wie er mit der heydenfchaft vor terbe crifteliche kraft Herre kvining dar ratet zv daz er icht an dem riche tv

[...] weren des teufe[...] Swelche der helfe fich br[...] 21055 Die folt ir haben vor heyden vnde von den louben gefcheyden vnde betalle han ir gůt Ich bin der daz gerne tůt Sprach der romare loys

21060 Willehalm ${ }^{7}$ der cronen pris were ich biz ich tot gelige Oder gar behaben den fige Ich getrůwe winnen eyn her daz terremer her vber mer 21065 So grozez niember bringet Groz leyt min herze twinget 


\section{Versoseite:}

$[\ldots]$ herzen $g[\ldots]$

[...] ionge kvining $[. . .]^{8}$

ez ift rechtes kvininges mvit

[...] ir weret des riches gůt

$21095 \mathrm{Ob}$ ir nicht des riches hettet

Swa ir willehalme helfe tettet des folt ir vch cleyne fchamen Sin fwefter von $\mathrm{v}$ hat den namen Daz fie heyzet eyn kv̊ningin

2 IIOO Malifer la dine rede fin wizze vůr war ich bringe eyn her kvimet terremer vber mer daz im die vart gerůwet Min ftete die fi ver trůwet

$\mathrm{Die}^{9}$ kv̊ningin an ir brůder fach zv dem fie vil fưze ßprach wizze daz ich dich minne

Mit herze vnde mit finne vnde bedarf der koning foldes Ich gebe durch dich goldes Daz ez in dvnket gar zv vil Gebe ers fwer im dienen wil

21135 wir solen da vor nicht gutes Parn Ich wil mit den kvoninge varn vnde fehen din reynes wip wol ir daz ir kvfche lip Ift an lobe alfo breyt 2II40 kvmber den ir herze treyt

Auf Grundlage von Hübners Textausgabe lässt sich rekonstruieren, dass zwischen den im Fragment überlieferten Textpassagen zweimal 24 und einmal 22 Verse fehlen (Abb. 3). ${ }^{\text {.0 }}$ Die ursprüngliche Handschrift muss demnach 36 oder 38 Zeilen pro Spalte aufgewiesen haben. Anhand der Abmessungen der Zeilen lässt sich zudem die ursprüngliche Blattgröße zumindest näherungsweise bestimmen. Nimmt man 38 Zeilen à $7 \mathrm{~mm}$ an, ergibt sich eine Gesamtblatthöhe von mindestens $318 \mathrm{~mm}$, wobei ein oberer Blattrand unbekannter Höhe hinzukäme - bei $52 \mathrm{~mm}$ wie unten, wäre demnach mit ca. $370 \mathrm{~mm} \mathrm{zu} \mathrm{rech-}$ nen. Für den Schriftraum ergäbe sich bei 38 Zeilen eine Höhe von $266 \mathrm{~mm}$.

Bei der Schrift handelt es sich um eine sauber ausgeführte Textualis. Auffällig ist vor allem das doppelstöckige $a$, das zwar teils schon in der zweiten Hälfte des i3. Jahrhunderts in der Textualis auftritt, vor allem aber im I4. Jahrhundert Anwendung findet und in dem Fragment durchgängig geschrieben wird. Signifikant sind darüber hinaus die zahlreichen Haarstriche, die vermehrt seit dem dritten Viertel des I3. Jahrhunderts auftreten. In der vorliegenden Handschrift finden sie sich nicht nur am Wortende, sondern auch beim $z$. Bei dem $z$ handelt es sich zudem nicht um ein geschwänztes $z$, was ebenfalls in das beginnende I4. Jahrhundert deutet. Die Buchstabenbrechungen sind insgesamt nicht besonders stark ausgeprägt und es finden sich zwar Buchstabenverbindungen bei $d e$, aber noch keine Ligaturen bei $b e$ oder $b o$. Nach $b, o, p$ und $v$ notiert der Schreiber ein rundes $r$, innerhalb der $t t$-Ligaturen wird der Schaft des zweiten $t$ überhöht und als Schluss-s findet sich häufig das sogenannte Brezel-s, welches zudem über das Mittelband hinausragt allesamt Merkmale einer Textualis des I4. Jahrhunderts. Jedoch fehlen im vorliegenden Text noch die $i$-Punkte, welche sich vor allem nach dem zweiten Viertel des I4. Jahrhunderts durchsetzen. Auf Grundlage dieser Charakteristika kann man die Schrift und das Fragment folglich in die erste Hälfte des I4. Jahrhunderts datieren. ${ }^{\text {II }}$

Einen wichtigen Hinweis auf den Werkzusammenhang des Fragments bietet darüber hinaus die auf der Versoseite des Fragments notierte Kustode xxviij. Hätte der ursprüngliche Codex lediglich den ,Rennewart' umfasst, müssten 21.00o Verse auf 28 Lagen verteilt sein. Bei 38 Zeilen pro Spalte wären das 152 Zeilen pro Blatt - je 76 auf der Vorder- und Rückseite. Bei 152 Versen pro Blatt hätte der Schreiber also bis zum 2I.000. Vers ca. I38 Blätter benötigt. Versucht man jedoch die Anzahl der Blätter pro Lage zu errechnen und teilt hierfür die 138 Blätter durch 28 Lagen, ergeben sich aufgerundet fünf Blätter. Da eine Lage 
allerdings jeweils aus ineinandergelegten Doppelblättern besteht, ist eine ungerade Zahl eher unwahrscheinlich. Hieraus kann man schlussfolgern, dass im Ursprungscodex vermutlich noch ein oder mehrere Texte vor dem ,Rennewart' gestanden haben dürften. Da der ,Rennewart' häufig als dritter Teil des, Willehalm'-Zyklus überliefert ist $^{12}$, erschien eine entsprechende Beispielrechnung lohnenswert. Addiert man die 9.700 Verse der ,Arabel' mit den I4.00o des ,Willehalm' und den 2I.000 des ,Rennewart' bis zum Fragmentblatt, kommt man zusammen auf 44.700 Verse. Diese geteilt durch 152 Verse pro Blatt ergeben ca. 294 Blätter, welche man wiederum durch 28 Lagen teilt, wodurch sich ca. I0,5 Blätter pro Lage errechnen lassen. Folglich hätten die Lagen eines die gesamte, Willehalm'-Trilogie umfassenden Codex mit den formalen Charakteristika des Fragments jeweils fünf oder sechs Doppelblätter umfasst, was keineswegs abwegig erscheint. ${ }^{13}$

Ein Abgleich mit den bisher bekannten Textzeugen durfte sich mithin nicht auf die überlieferten Handschriften des ,Rennewart' beschränken, sondern musste darüber hinaus alle Codices discissi der ,Arabel' und des ,Willehalm' einschließen. Und tatsächlich war es möglich, das Heringer ,Rennewart'-Fragment einem bereits bekannten Textzeugen zuzuordnen.

In der Gottfried Wilhelm Leibniz Bibliothek Hannover wird unter der Signatur Ms. IV 489 ein Codex verwahrt, der auf I5I Blättern zwei ursprünglich selbstständige Handschriften in sich vereint. ${ }^{14}$ Wohl bereits im 15 . Jahrhundert wurden Ulrichs von dem Türlin ,Arabel' (Bl. Ir-65v) und Albrechts ,Jüngerer Titurel' (B1. 66r-I5ov) zusammengebunden, wobei sich in beiden Fällen lediglich Fragmente erhalten haben. Der ursprüngliche Holzdeckeleinband mit Lederbezug, Streicheisenlinien und Blindstempeln, den Werner Wolf $1955^{\text {15 }}$ beschrieb, wurde 1965 infolge eines Wasserschadens durch einen modernen Lederbezug mit Messingschließen ersetzt und gilt seitdem mitsamt der im Spiegel eingeklebten lateinischen Urkunde als verschollen. Da hierdurch auch die alte Signatur „I.78“ und die in das Pergament eingeschnittenen Buchstaben „HMVN“verlorengingen, lassen sich kaum Aussagen hinsichtlich der Provenienz des Codex treffen. ${ }^{16}$ Belegt ist lediglich, dass Christian Ludwig Scheidt den Band wohl am 20. Dezember 1749 von einem Hofrat von Gemmingen für die Königliche Bibliothek erworben hat. ${ }^{17}$

Der erste Teil des Manuskripts stimmt in allen formalen Merkmalen mit dem Heringer Fragment überein: Jedes Pergamentblatt, welches $320 \times 220 \mathrm{~mm}$ misst, umfasst auf einem Schriftraum von $260 \times 170 \mathrm{~mm} 38$ Verse in zwei Spalten. Der Schreiber ist zweifelsfrei mit demjenigen des Heringer Fragments identisch. Mehrere Risse im Beschreibstoff wurden vernäht. Wie im Fragment des ,Rennewart' sind auch in der Hannoveraner Handschrift die Versanfänge nicht abgesetzt und es finden sich zweizeilige rubrizierte Lombarden am Anfang der Strophen. Darüber hinaus machen entsprechende Freiräume deutlich, dass der Codex mit einer nicht ausgeführten zwölfzeiligen Anfangsinitiale und mehreren Illustrationen versehen werden sollte, für die sich bereits rubrizierte Beschreibungstexte finden. Die auf den Versoseiten der letzten Blätter einer Lage notierten Kustoden belegen zudem die Annahme, dass der Ursprungscodex mit der ,Arabel' einsetzte und neben dem ,Rennewart wohl auch den, Willehalm' umfasst haben dürfte. ${ }^{18}$ Der Verbleib der fehlenden Seiten ist jedoch nach wie vor unklar. Auch konnte bislang nicht geklärt werden, wie das ,Rennewart'-Fragment nach Heringen (Helme) gelangte, sodass weitergehende Provenienzrecherchen wünschenswert erscheinen.

In jedem Fall liegt durch den Fragmentfund nun aber ein neues Textzeugnis vor, das nicht nur für Studien zum ,Rennewart' Ulrichs von Türheim, sondern darüber hinaus für die ,Willehalm'-Forschung interessant sein dürfte. Und da der Bürgermeister von Heringen (Helme) in Reaktion auf den Fund einer Verbringung der gesamten historischen Registratur 
der Gemeinde in das Kreisarchiv Nordhausen für deren Zugänglichkeit gesorgt, sodass weitere zugestimmt hat, ist in Zukunft nicht nur für die Forschungen jederzeit angestellt werden können. Erhaltung dieser wichtigen Quelle, sondern auch

\section{Kontakt}

Jessica Bruns $\cdot$ Christian Speer

Martin-Luther-Universität Halle-Wittenberg • Institut für Geschichte · DFG-Projekt ,Index Librorum Civitatum

Emil-Abderhalden-Str. 26-27 · 06099 Halle (Saale)

E-Mail: jessica.back@geschichte.uni-halle.de·christian.speer@geschichte.uni-halle.de

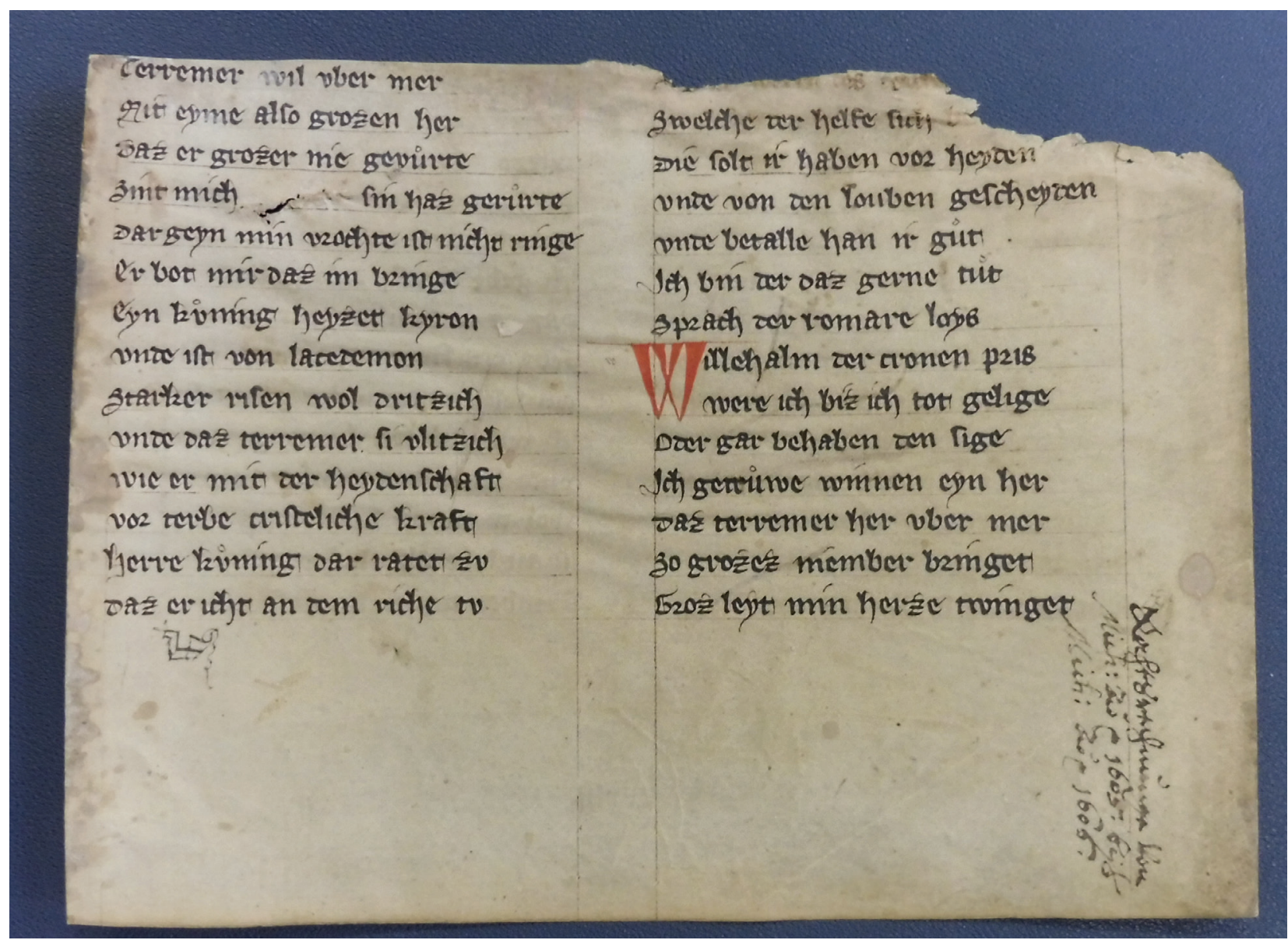

Abb. r: Rectoseite des Fragments 


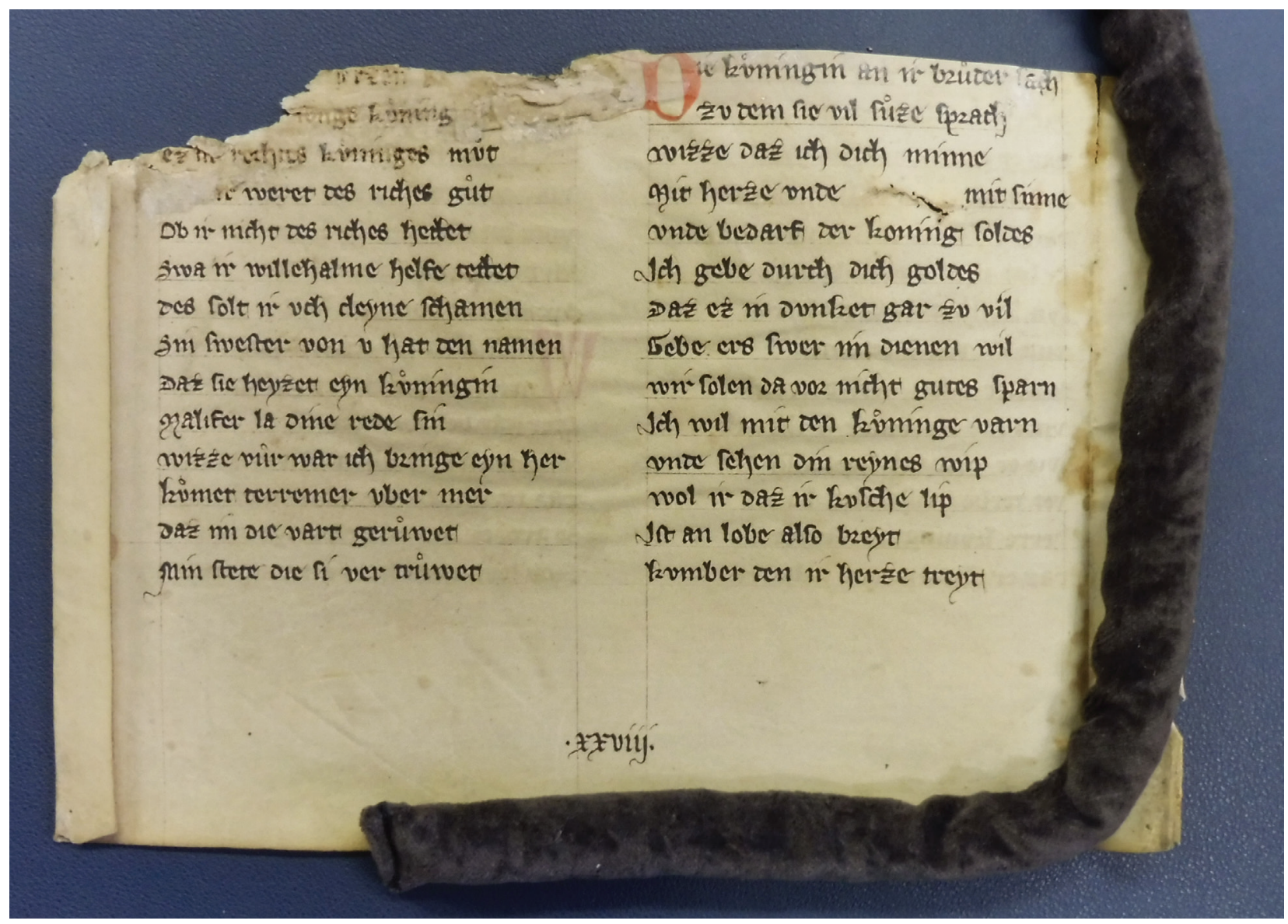

Abb. 2: Versoseite des Fragments
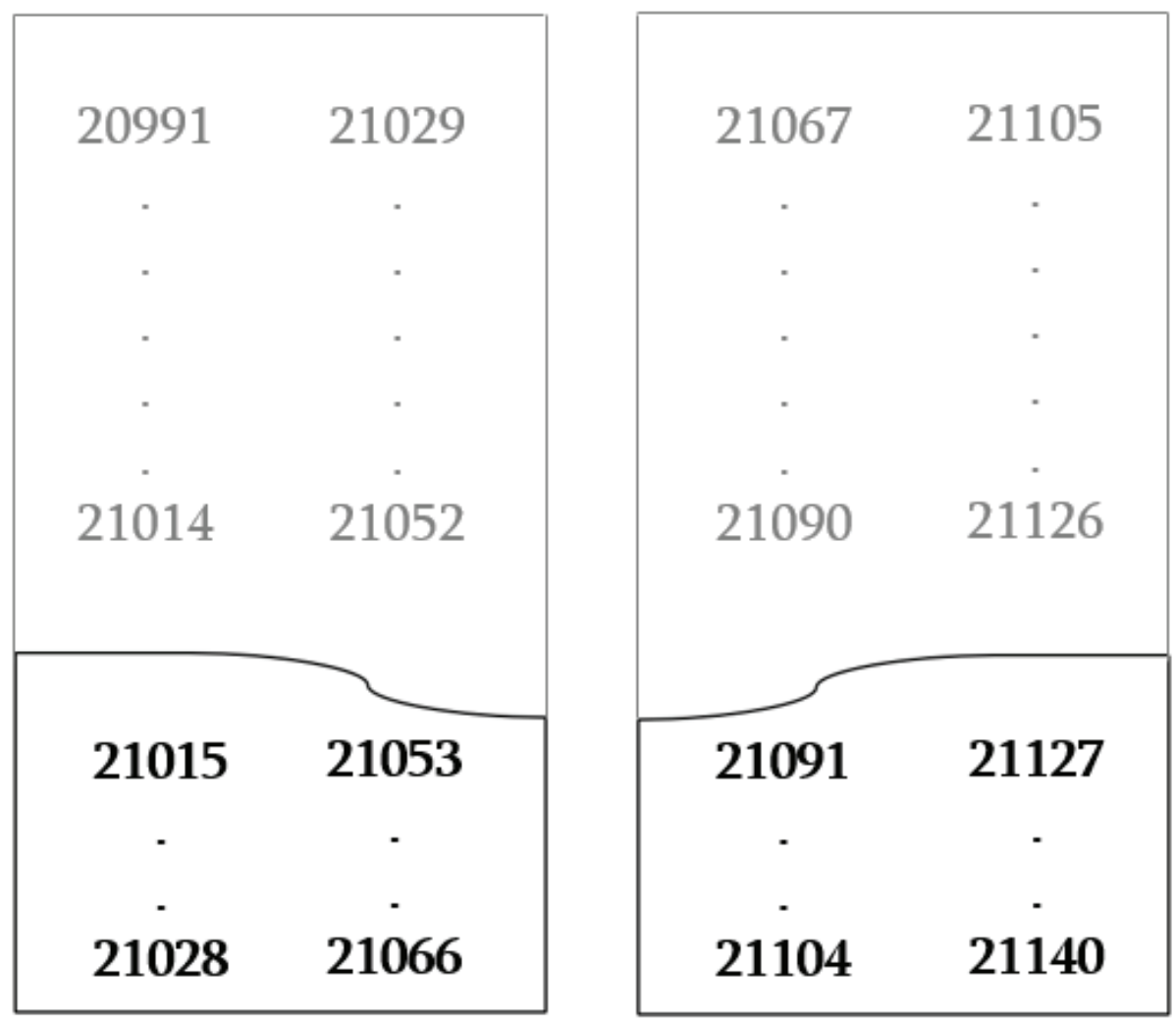

Abb. 3: Schematische Darstellung zum Versbestand des Fragments 


\section{Anmerkungen}

I Siehe zur Datenbank und Projektbeschreibung <https://www.stadtbuecher.de/de/about/> sowie zu den Stadtbuchbeständen von Heringen (Helme) <https://www.stadtbuecher.de/de/stadtbuecher/deutschland/thueringen/heringen-helme/> (8.4.202I).

2 Vgl. zum Beispiel im ,Gesamtkatalog der Wiegendrucke' die in den Einband eines Zwickauer Stadtbuches eingeklebten Blätter eines Prognostikons von 1490 sowie Ulrich-Dieter Oppitz, Stadtbücher- und Fragmentenforschung. Texte von Rechtsbüchern in Eilenburg, Görlitz und Pößneck, in: Neues Archiv für Sächsische Geschichte 85 (2014), S. 226-236.

3 Wilhelm Schlüter, Die Rechnungsbücher des Rates der Stadt Heringen im Kreisarchiv Nordhausen, in: Mitteldeutsche Familienkunde 33 (1992), Band io, Heft 4, S. 358-372.

4 Hermann Hiller schreibt 1939 in seinem Bericht an die Archivberatungsstelle der Provinz Sachsen: „Die alten Ratsrechnungen von I59I-I660 sind in II stattlichen Bänden in Sackleinen auf Kosten der Stadt dauerhaft eingebunden worden, dazu noch 5 Bände Reisepässe, Testamente etc. Der Bürgermeister, der ein großes Interesse für meine Arbeit zeigt, hat mir auch für die Zukunft Mittel zum Einbinden von jährlich etwa 8-Io Bänden zur Verfügung gestellt.“ Vgl. Landesarchiv Sachsen-Anhalt, Magdeburg, C 96 II, Nr. 23, B1. 3. Im Jahresbericht von I943 weist Hiller abermals darauf hin, dass „mehrere wertvolle Archivalien“ durch einen Buchbinder eingebunden worden seien, vgl. ebd. B1. 2r4. Für den wertvollen Hinweis auf diese Berichte sei Björn Schmalz herzlich gedankt.

$5 \mathrm{Zu}$ Ulrich von Türheim vgl. beispielsweise Peter Strohschneider, Ulrich von Türheim, in: ${ }^{2}$ VL Io (I999), Sp. 28-39, oder Eberhard Kurt Busse, Ulrich von Türheim (Palaestra I2I), Berlin I9I3. Die Textausgabe des, Rennewart' stammt aus dem Jahr 1938: Alfred Hübner (Hg.), Ulrich von Türheim: Rennewart. Aus der Berliner und Heidelberger Handschrift (Deutsche Texte des Mittelalters 39), Berlin 1938 (Nachdruck Berlin/Zürich I966). Zu den bekannten ,Rennewart'-Handschriften vgl. Klaus Klein, Neues Gesamtverzeichnis der Handschriften des ,Rennewart' Ulrichs von Türheim, in: Wolfram-Studien XV (I998), S. 45I-493. Zu späteren Fragmentfunden vgl. Christoph Fasbender, Jenaer Bruchstück einer unbekannten ,Rennewart'-Handschrift, in: ZfdA I34 (2005), S. I86-I90; Annelen Ottermann und Klaus Klein, Ein unbekanntes ,Rennewart'-Fragment in Mainz, in: ZfdA I37 (2008), S. 37I-376; Elisabeth Wunderle, Ein ,Rennewart'-Fragment in der Studienbibliothek Dillingen, in: ZfdA I42 (20I3), S. 8I-85 sowie die Auflistung von ,Rennewart'-Handschriften unter <https://handschriftencensus.de/werke/388> (8.4.202I).

6 Vgl. Hübner [Anm. 5], S. 307-309.

$7 \quad W$ als zweizeilige rote Lombarde.

8 Es wäre auch die Lesart iunge denkbar.

$9 D$ als zweizeilige rote Lombarde.

Io Vgl. Hübner [Anm. 5], S. 308f. Dass einmal lediglich 22 Verse fehlen, könnte damit zusammenhängen, dass einige der Verse 2IIO5-2II27 besonders lang sind. Doch auch eine gegenüber Hübner abweichende Textfassung oder Aussparungen für Schmuck- oder Gliederungselemente können nicht ausgeschlossen werden.

II $\mathrm{Zu}$ den angeführten Schriftmerkmalen und deren Datierung vgl. Karin Schneider, Paläographie und Handschriftenkunde für Germanisten. Eine Einführung, 3., überarbeitete Auflage (Sammlung kurzer Grammatiken germanischer Dialekte B. Ergänzungsreihe Nr. 8), Berlin/Boston 20I4, S. 42-50.

12 So etwa in Berlin, Staatsbibliothek, mgf I063; Bibliotheca Bodmeriana, Cod. Bodm. I70; Heidelberg, Universitätsbibliothek, Cpg 404; Kassel, Universitätsbibliothek, LMB, $2^{\circ}$ Ms. poet. et roman. I; Köln, Historisches Archiv der Stadt, Best. 7010 (W) 355; Wien, Österreichische Nationalbibliothek, Cod. 3035; sowie Cod. Ser. nova 2643; Wolfenbüttel, Herzog August Bibliothek, Cod. 30.12 Aug. $2^{\circ}$ oder in den an mehreren Orten fragmentarisch überlieferten Handschriften <https://handschriftencensus.de/II3I>, <https://handschriftencensus.de/I786> und $<$ https://handschriftencensus.de/I785> (8.4.202I) 
I3 Herzlich zu danken ist an dieser Stelle Daniel Könitz und den übrigen Mitarbeitern der Arbeitsstelle ,Handschriftencensus', die uns nicht nur tatkräftig beim Abgleich des Heringer-Fragments mit anderen Textzeugen unterstützt, sondern darüber hinaus auch die beiden obenstehenden Beispielrechnungen angeregt haben.

I4 Für die Beschreibungen der Handschrift vgl. Eduard Bodemann, Die Handschriften der Königlichen Öffentlichen Bibliothek zu Hannover, Hannover I867, S. 83-85 (Nr. 489); Werner Wolf (Hg.), Albrechts von Scharfenberg Jüngerer Titurel, Bd. I (Strophe I-I957). Nach den ältesten und besten Handschriften (Deutsche Texte des Mittelalters 45), Berlin 1955, S. LVIf. (Nr. 3); Peter Jörg Becker, Handschriften und Frühdrucke mittelhochdeutscher Epen. Eneide, Tristrant, Tristan, Erec, Iwein, Parzival, Willehalm, Jüngerer Titurel, Nibelungenlied und ihre Reproduktion und Rezeption im späteren Mittelalter und in der frühen Neuzeit, Wiesbaden I977, S. I22f. (Nr. 3); Betty C. Bushey, Neues Gesamtverzeichnis der Handschriften der ,Arabel` Ulrichs von dem Türlin, in: Wolfram-Studien VII (1982), S. 228-286, hier S. 244f.; Helmar Härtel und Felix Ekowski, Handschriften der Niedersächsischen Landesbibliothek Hannover, 2. Teil: Ms I 176a-Ms Noviss. 64 (Mittelalterliche Handschriften in Niedersachsen 6), Wiesbaden I982, S. I4of.; Bernd Bastert, Helden als Heilige. Chanson de geste-Rezeption im deutschsprachigen Raum (Bibliotheca Germanica 54), Tübingen/Basel 20ı0, S. 470 und Klaus Klein, Beschreibendes Verzeichnis der Handschriften (Wolfram und Wolfram-Fortsetzer), in: Joachim Heinzle (Hg.), Wolfram von Eschenbach. Ein Handbuch, Berlin/Boston 20II, Bd. 2, S. 94I-IO02, hier S. 96I und 990 sowie den Eintrag im ,Handschriftencensus' unter <https://handschriftencensus.de/3640> (8.4.202I).

I5 Vgl. Wolf [Anm. I4], S. LVI.

I6 Als Entstehungsraum geben Härtel und Ekowski für den ,Arabel'-Teil Südwestdeutschland an. Die Schreibsprache beschreiben sie als Mittelhochdeutsch mit alemannischem Einschlag, vgl. Härtel/Ekowski [Anm. I4], S. I4O. Wolf geht hingegen von einer niederdeutsch geprägten Abschrift einer bairischen Vorlage aus, vgl. Wolf [Anm. I4], S. LVII.

17 Vgl. Härtel/Ekowski [Anm. I4], S. I40, die eine Kaufsumme von 30 Talern angeben. Bei Bushey [Anm. I4], S. 244 werden 1749 als Erwerbungsjahr, aber 24 Taler als Preis angeführt. Bodemann [Anm. I4], S. 85 nennt hingegen I750 als Erwerbungsjahr und weist Kosten von 24 Talern aus. Als für den Erwerbsvorgang einschlägige Quellen konnten wir die im Niedersächsischen Landesarchiv, Abteilung Hannover, verwahrten Akten NLA HA Dep. I03 XXXV Nr. 159 sowie NLA HA Dep. I03 XXXV Nr. 1583 identifizieren. Obwohl eine Genehmigung zur Einsichtnahme in diese gesperrten Akten des Königlichen Hausarchivs vorlag, war es aufgrund der coronabedingten Archivschließungen und Dienstreisebeschränkungen nicht mehr möglich, die Archivalien vor Redaktionsschluss zu prüfen. Hinsichtlich der Identität des Hofrats von Gemmingen wäre aus unserer Sicht Eberhard Friedrich Freiherr von Gemmingen (I726-I79I) denkbar, der ab I748 württembergischer Regierungsrat war und eine umfangreiche Bibliothek sowie ein ausgeprägtes Interesse an mittelalterlicher Literatur besaß. Doch auch Philipp Freiherr von Gemmingen (I728-I800) oder Ludwig Freiherr von Gemmingen (Wolfenbüttler Hofrat und I740-I764 Oberappelationsgerichts-Vizepräsident) kämen in Frage.

I8 Auf folgenden Blättern finden sich Kustoden: Bl. I4v: ii, Bl. 37v: v, Bl. 44v: vj und Bl. 59v: viij. Härtel/Ekowski [Anm. I4], S. I40 geben als Lagenformel an: IV(8). IV-2(I4). IV(22). VI-I(29). IV(37). IV-I(44). IV-I(5I). IV(59). $\mathrm{I}(6 \mathrm{I})$. II(65). 